\title{
MKS System
}

National Cancer Institute

\section{Source}

National Cancer Institute. MKS System. NCI Thesaurus. Code C42563.

The cluster of metric units based on the meter, the kilogram, and the second as base units. 